\title{
Relationships between clozapine and norclozapine plasma concentrations, clozapine dose, and clinical response in Tunisian patients with schizophrenia-treatment resistance
}

\author{
Aida Taieb ${ }^{1,2 *}$, Fatma B'chir ${ }^{3}$, Roland Molinié ${ }^{1}$, José-Edmundo Nava-Saucedo ${ }^{1}$, Jaafer Nakhli ${ }^{4}$, \\ Marc-André Fliniaux ${ }^{1}$, Bechir Ben Hadj $\mathrm{Ali}^{4}$, Saad Saguem ${ }^{2}$ \\ ${ }^{1}$ Unit of Plant Biology and Insect Pests, University of Picardy Jules Verne, Amiens, France \\ ${ }^{2}$ Metabolic Biophysics and Applied Pharmacology Laboratory, Department of Biophysics, Medicine Faculty of Sousse, Sousse, Tunisia \\ ${ }^{3}$ Laboratory of Natural Substances, National Institute of Research and Physical Chemical Analysis, Technopole Sidi Thabet, Tunis, \\ Tunisia \\ ${ }^{4}$ Psychiatry Department of Central Hospital University (CHU), Sousse, Tunisia \\ Email: ${ }^{*}$ aida.taieb@u-picardie.fr, bchirfatma@hotmail.fr
}

Received 20 April 2012; revised 26 May 2012; accepted 7 June 2012

\begin{abstract}
The present study investigated relationships between clozapine dose, clozapine and norclozapine plasma concentrations, and clinical responses to clozapine treatment in Tunisian schizophrenics. Fourteen schizophrenia-treatment resistant patients, recruited for this study, were treated with clozapine for 45 days. Patient health improvement was assessed before and after each cycle of two weeks of clozapine therapy, using the Brief Psychiatric Rating Scale (BPRS). Plasma clozapine and norclozapine concentrations were determined by high-performance liquid chromatography (HPLC). No significant correlations between plasma clozapine and norclozapine concentrations and clinical health improvement among our schizophrenic patients were found. However, a significant correlation was observed between clinical health improvement given by BPRS scores and norclozapine plasma concentration to daily clozapine dose ratio (NCZ/D). Despite the small sample size of our study, our findings suggest that the clozapine therapy response variations observed in our patients may be, in part, explained by the interindividual differences in plasma norclozapine concentration to clozapine dose ratio (NCZ/D). So the NCZ/D parameter could be used as a good indicator for adjusting the clozapine dose-adaptation strategy and consequently for improving the clinical psychopathological state of schizophrenia-treatment resistant patients.
\end{abstract}

Keywords: Clozapine; Norclozapine; HPLC; Tunisian Schizophrenic; BPRS

\footnotetext{
${ }^{*}$ Corresponding author.
}

\section{INTRODUCTION}

Clozapine (CZ) is a second-generation antipsychotic drug. It is primarily used in the treatment of schizophreniatreatment resistant psychopathology. However, treatment with clozapine is associated with several side effects that complicate the use of the drug and necessitate therapeutic monitoring $[1,2]$. This monitoring allows the optimization of the therapeutic dose-response efficacy and the prevention of excessive toxicity of $\mathrm{CZ}$, reducing in this way the risk of relapse [3-5]. Despite the effectiveness of clozapine as a standard drug for schizophrenia-treatment resistance, important individual variations in the response to clozapine therapy were reported $[6,7]$.

The mechanism underlying the differences in clozapine treatment response is not well known. Some studies attribute these differences to patients' sex, age, body weight, and physiology [8]. Other studies suggest that these variations are related to the plasma concentrations of clozapine and its active metabolite, norclozapine [9], with the explanation that monitoring plasma clozapine concentrations may play a useful role in the management of patients with schizophrenia. Moreover, it may be possible that the metabolic polymorphism of clozapine elimination, attributed to genetic and/or environmental origins, modifies clozapine plasma concentration and elimination and thus its psychotherapeutic effects.

Therefore, this study has been undertaken to explore possible correlations between clinical responses to clozapine and parameters such as clozapine and norclozapine plasma concentrations, clozapine clearance, clozapine metabolic index, and norclozapine plasma concentration to daily clozapine dose ratio. Our interest is in offering some explanations for the interindividual variability of 
clozapine therapy response.

\section{MATERIALS AND METHODS}

\subsection{Patients}

Fourteen patients with schizophrenia-treatment resistance were recruited for this study. These patients were composed of 3 males and 11 females aged 19-62 years and were taken from the psychiatric department of the University Hospital Centre (CHU) Farhat Hached of Sousse (Tunisia). The diagnosis of schizophrenia-treatment resistance was established by the DSM-IV (Diagnostic and Statistical Manual of Mental Disorders) criteria for schizophrenia [10]. The physical characteristics of the patients are given in Table 1. All the patients in this study were treated with $\mathrm{CZ}$, and they did not take any other associated treatment.

The fourteen patients selected for this study represent $35 \%$ of the total number of cases treated in the hospital of Sousse and $14 \%$ of the total cases of schizophreniatreatment resistance in Tunisia. The population of this country is over one million inhabitants. Treatment-resistant schizophrenics represent only $0.01 \%$ of the population. Thus, the fourteen patients recruited to this study are enough representatives for a local-level study, but not for a global approach.

This study was carried out in the biophysics laboratory at the Faculty of Medicine. The ethics committee of the Faculty of Medicine approved the study, and each patient provided informed consent.

\subsection{Clinical State Assessment}

The effectiveness of treatment for the psychopathological state was determined by the Brief Psychiatric Rating Scale (BPRS). The BPRS estimations obtained before the treatment with $\mathrm{CZ}$ and during the therapy period were reported in our study as health improvements and expressed in percentages. A BPRS value is determined before the application of the treatment and a new BPRS value is determined after the treatment (45 days later). The final BPRS value is given by the equation: ([BPRS before treatment-BPRS after treatment]/BPRS before treatment) $\times 100[11]$.

\subsection{HPLC Plasma Analysis and Clozapine and Norclozapine Concentrations}

The schizophrenic patients recruited into the study were given clozapine at a dosage of 100 - $900 \mathrm{mg} /$ day for 45 days. Blood samples were drawn from each patient 12 hours after the last intake of the CZ dosage at the end of each cycle of 15 days over a period of 45 days.

Blood samples were immediately centrifuged at 1800 g for 15 minutes at $4^{\circ} \mathrm{C}$, and the plasma samples collected
Table 1. Physical characteristics of 14 schizophrenic patients.

\begin{tabular}{ccccc}
\hline Patient & Gender & Age (years) & Weight (Kg) & Height (m) \\
\hline (a) & W & 35 & 60 & 1.58 \\
(b) & W & 39 & 60 & 1.60 \\
(c) & W & 43 & 70 & 1.69 \\
(d) & M & 40 & 95 & 1.77 \\
(e) & W & 42 & 88 & 1.75 \\
(f) & W & 62 & 76 & 1.65 \\
(g) & M & 19 & 69 & 1.70 \\
(h) & M & 33 & 83 & 1.73 \\
(i) & W & 22 & 70 & 1.62 \\
(j) & W & 23 & 74 & 1.65 \\
(k) & W & 19 & 62 & 1.65 \\
(l) & W & 48 & 80 & 1.65 \\
(m) & W & 40 & 62 & 1.62 \\
(n) & W & 29 & 62 & 1.65 \\
\hline
\end{tabular}

were stored at $-20^{\circ} \mathrm{C}$ until the time of analysis. The concentrations of $\mathrm{CZ}$ and NCZ were assessed by the HighPerformance Liquid Chromatography (HPLC) method developed by Shen et al. [12], with some modifications.

The separation of these analytes was performed using reverse phase HPLC (Agilent 1200). A volume of $5 \mu \mathrm{L}$ of prepared plasma was injected into the analytical column (C18 ODS, Agilent type) maintained at a temperature of $30^{\circ} \mathrm{C}$. The elution was maintained in isocratic mode with a mobile phase consisting of water, acetonitrile, and N,N-dimethyloctylamine (62.6:37:0.4). The elution flow rate was $1 \mathrm{~mL} \cdot \mathrm{min}^{-1}$. The analytes were detected at a wavelength of $280 \mathrm{~nm}$. Plasma clozapine and norclozapine concentrations were calculated by Chemstation software using preestablished calibration curves.

\subsection{Measurement of Clozapine Metabolic Ratio}

Norclozapine is the main metabolite of clozapine. The metabolic pathway from clozapine to norclozapine is mediated mainly by the hepatic CYP1A2 enzyme.

The measurement of the concentration ratio of norclozapine to clozapine (NCZ/CZ) was used as a metabolic marker to evaluate CYP1A2 activity and therefore the elimination profile of CZ.

\subsection{Measurement of Clozapine Clearance}

Clozapine clearance was determined by the ratio of clozapine dose (D) intake to clozapine plasma concentration (D/CZ).

\subsection{Statistical Analysis}

Regression analysis was used to find correlations be- 
tween the different parameters studied and the BPRS scores (health improvement of patients). Analyses of variance (ANOVA) and t-tests were used to test correlations between clozapine and norclozapine plasma concentrations, clozapine metabolic index, and norclozapine plasma concentration to daily clozapine dose ratio as well as percent improvement in BPRS scores.

\section{RESULTS}

\subsection{Association between CZ and NCZ Plasma Concentrations, CZ Clearance, and BPRS Score}

The plasma concentrations of CZ and NCZ were determined for a period of 45 days at the end of each twoweek cycle. The mean values of CZ and NCZ plasma concentrations $( \pm 90 \%$ confidence intervals), CZ clearance, and clozapine dose intake for each patient as well as percentage BPRS scores are given in Table 2.

There is large intervariability in CZ mean plasma concentrations, ranging from 120 to $1304 \mathrm{ng} \cdot \mathrm{mL}^{-1}$ for all patients. This wide variation was also observed for the NCZ mean plasma concentrations.

The CZ plasma concentration parameter, which is used as an indicator of the treatment response and dose adaptation, shows no significant correlation with the health state improvement given by BPRS scores $(r=0.0989$; $p<$ 0.737) (Figure 1).

For example, patient (a) who presents with severe toxicity (agranulocytosis) has a BPRS value of $7.3 \%$ and a CZ plasma concentration measure of $1280 \mathrm{ng} \cdot \mathrm{mL}^{-1}$, whereas similar levels of plasma $\mathrm{CZ}$ concentration, such

Table 2. CZ, NCZ, D/CZ values and BPRS scores of 14 schizophrenic patients.

\begin{tabular}{cccccc}
\hline Patient & $\mathbf{C Z}(\mathbf{n g} / \mathbf{m L})$ & $\begin{array}{c}\mathbf{N C Z} \\
(\mathbf{n g} / \mathbf{m L})\end{array}$ & $\mathbf{D}(\mathbf{m g} / \mathbf{d a y})$ & $\begin{array}{c}\mathbf{D} / \mathbf{C Z} \\
\text { (L/h) }\end{array}$ & $\begin{array}{c}\text { BPRS } \\
\text { (\%) }\end{array}$ \\
\hline (a) & $1280 \pm 292$ & $42 \pm 10$ & 400 & 13 & 7.3 \\
(b) & $120 \pm 27$ & $27 \pm 4$ & 300 & 104.2 & 18.5 \\
(c) & $1203 \pm 198$ & $127 \pm 39$ & 400 & 13.9 & 20 \\
(d) & $843 \pm 492$ & $228 \pm 76$ & 900 & 44.5 & 22.9 \\
(e) & $710 \pm 695$ & $123 \pm 85$ & 400 & 23.5 & 26.9 \\
(f) & $120 \pm 20$ & $36 \pm 11$ & 100 & 34.7 & 31.9 \\
(g) & $495 \pm 51$ & $173 \pm 43$ & 650 & 54.7 & 32.5 \\
(h) & $499 \pm 121$ & $145 \pm 46$ & 500 & 41.8 & 35.6 \\
(i) & $1304 \pm 784$ & $214 \pm 91$ & 550 & 17.6 & 42.4 \\
(j) & $1080 \pm 330$ & $194 \pm 34$ & 500 & 19.3 & 50 \\
(k) & $618 \pm 679$ & $140 \pm 19$ & 400 & 27 & 53 \\
(l) & $650 \pm 63$ & $150 \pm 28$ & 400 & 25.6 & 54.2 \\
(m) & $1145 \pm 172$ & $158 \pm 28$ & 400 & 14.6 & 54.9 \\
(n) & $950 \pm 73$ & $181 \pm 29$ & 500 & 21.9 & 68.8 \\
\hline
\end{tabular}

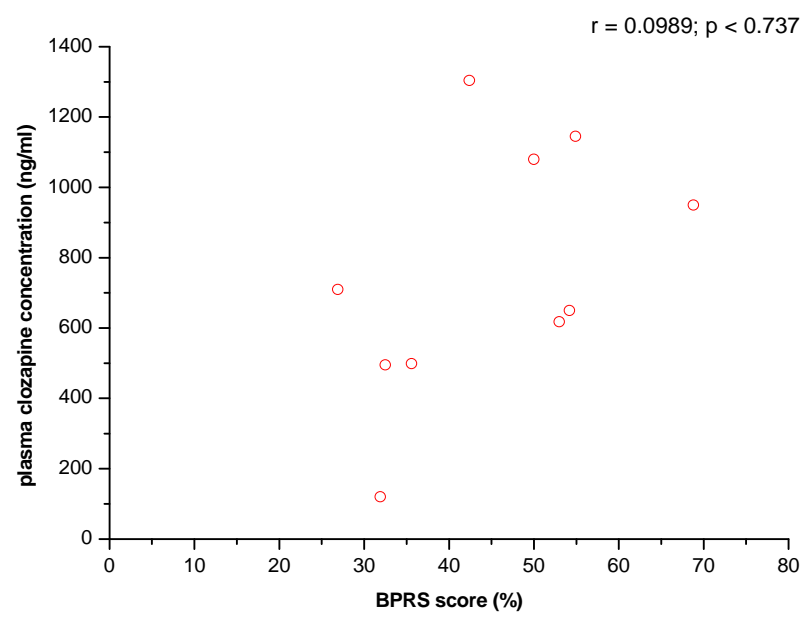

Figure 1. Relationship between BPRS scores and plasma levels of clozapine.

as 1304 and $1145 \mathrm{ng} \cdot \mathrm{mL}^{-1}$, were found in patients (i) and (m) who had higher BPRS values of $42.4 \%$ and $54.9 \%$, respectively.

Furthermore, although the BPRS values of $53 \%$ and $54.2 \%$, respectively, for patients $(\mathrm{k})$ and $(\mathrm{l})$ were found to be similar to those of patients (i) and (m), their corresponding plasma CZ concentrations of 618 and 650 $\mathrm{ng} \cdot \mathrm{mL}^{-1}$, respectively, were very low compared to those found in patients (i) and (m).

The estimated CZ plasma clearance values (D/CZ) ranged between 13 and $104.2 \mathrm{~L} \cdot \mathrm{h}^{-1}$. Statistical analysis, illustrated in Figure 2, showed no significant relationship between response to clozapine treatment, assessed by BPRS scores, and CZ plasma clearance $(r=0.32$; $\mathrm{p}<$ 0.265). Of the fourteen schizophrenic patients in the study, four had BPRS scores falling within a narrow range ( $50 \%$ to $54.9 \%$ ), whereas they showed a wide range of CZ plasma clearance values (14.6 to $27 \mathrm{~L} \cdot \mathrm{h}^{-1}$ ). Furthermore, patients (l) and (m), who had practically the same BPRS score (ca. 54.55\%), showed different clearance values of 25.6 and $14.6 \mathrm{~L} \cdot \mathrm{h}^{-1}$, respectively.

\subsection{Association between NCZ/CZ Ratio and BPRS Scores}

Table 3 shows wide variations for all patients in NCZ to $\mathrm{CZ}$ ratio, which expresses CYP1A2 activity, ranging from $3.3 \%$ to $35 \%$. These variations suggest wide interindividual differences in CYP1A2 activity.

Statistical analysis did not provide any significant association between the NCZ/CZ ratio and the BPRS values ( $r=0.167 ; \mathrm{p}<0.569$ ). For example, an approximate ratio value of $22.8 \%$ was found in patients (k) and (l) along with higher BPRS scores of $53 \%$ and $54.2 \%$, respectively, whereas the same ratio value was found in patient (b) who had a lower BPRS value of 18.5\%. 


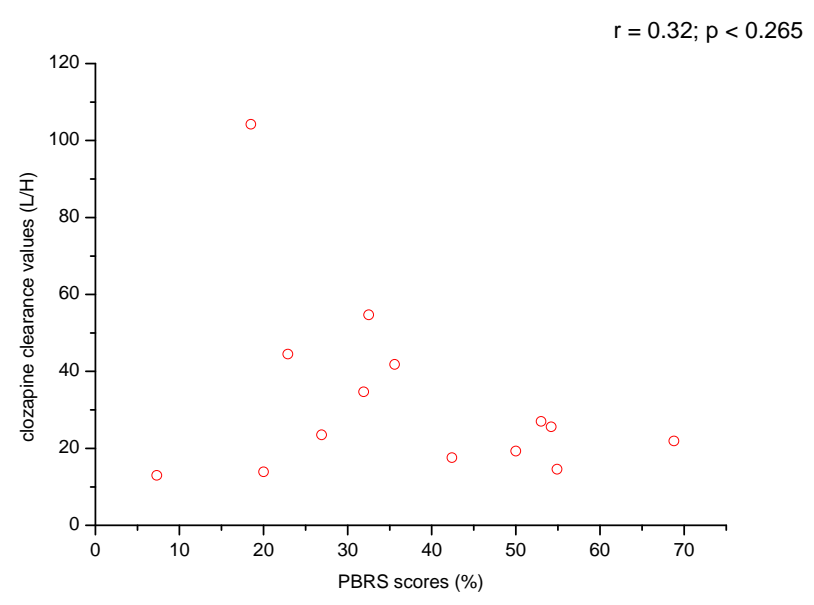

Figure 2. Relationship between BPRS scores and clozapine clearance levels.

Table 3. NCZ/CZ values and BPRS scores in 14 schizophrenic patients.

\begin{tabular}{ccc}
\hline Patient & NCZ/CZ (\%) & BPRS (\%) \\
\hline (a) & 3.3 & 7.3 \\
(b) & 22.5 & 18.5 \\
(c) & 10.6 & 20 \\
(d) & 27 & 22.9 \\
(e) & 17.3 & 26.9 \\
(f) & 30 & 31.9 \\
(g) & 35 & 32.5 \\
(h) & 29 & 35.6 \\
(i) & 16.4 & 42.4 \\
(j) & 18 & 50 \\
(k) & 22.6 & 53 \\
(l) & 23 & 54.2 \\
(m) & 13.8 & 54.9 \\
(n) & 19.1 & 68.8 \\
\hline
\end{tabular}

\subsection{Association between NCZ/CZ Ratio and BPRS Scores}

The NCZ plasma concentration to CZ dose ratio value (NCZ/D) was calculated for each patient. The ratios ranged from 7.8 to $34 \mathrm{~s} \cdot \mathrm{L}^{-1}$. There was a significant linear correlation ( $\mathrm{t}$ calculated value $=4.20 ; \mathrm{t}_{0.05}=2.18$ ) between the NCZ/D ratio and the BPRS levels ( $r=0.77$; $\mathrm{p}<0.0012$ ).

Figure 3 shows the estimated regression line $(y=12.3+$ $\left.0.38 x ; r^{2}=0.6\right)$ with the centroid (NCZ/D ratio and BPRS score means), the experimental values, the confidence band borders, and the prediction band borders. The prediction band is useful for making presumptions about a minimum BPRS level attained for a maximum NCZ/D ratio value.

\section{DISCUSSION}

In this preliminary study, we have tried to give some explanations for the large interindividual response variation to clozapine therapy observed in Tunisian schizophrenic patients.

We have noted that the improvement in the psychopathologic state of schizophrenic patients differs from one patient to another for the same clozapine dosage.

Multiple parameters have been measured in this study, as well as their relationships with the therapeutic effectiveness of CZ in schizophrenic patients.

The results show that no significant correlations were found between clinical health improvement in Tunisian schizophrenic patients and these measured parameters, namely plasma clozapine and norclozapine concentrations, clozapine metabolic ratio, and clozapine clearance. This result leads us to suggest that none of these parameters can be used as standard biomarkers for assessment of the therapeutic efficacy of clozapine [13,14].

Although there is considerable variability in daily drug treatment, a significant correlation between clinical health improvement, given by BPRS scores, and norclozapine plasma concentration to daily clozapine dose ratio (NCZ/D) was recorded. A wide variability in plasma CZ levels following intake of a large range of drug doses was observed in our patients. In fact, patients with a daily drug dose of $400 \mathrm{mg}$ showed higher plasma CZ concentrations than other patients taking a daily dose of $900 \mathrm{mg}$. So these differences in CZ plasma concentrations between patients may not be associated with differences in dose intake but rather with metabolic polymorphism under the influence of environmental and genetic factors $[15,16]$.

In this respect, particular attention has been focused on plasma drug concentration monitoring as a potential tool for determining the probability of achieving a satisfactory response [17]. However, there is controversy regarding the relationship between CZ plasma levels and psychopathologic state improvement. Some studies confirm an apparent relationship between plasma clozapine levels and its psychotherapeutic effect [6,9].

But other studies contradict these positive results [1722]. These findings are in close agreement with our results showing no association between plasma CZ concentration and amelioration of psychopathologic state in patients with CZ-treatment resistant schizophrenia.

Our findings in this study reported no significant relationships between clozapine metabolic ratio, clozapine clearance, and clinical health improvement in Tunisian schizophrenic-treatment resistant patients.

These results are consistent with those reported by Chong et al. and Doude van Troostwijk et al. [7,23], as are our results on the potential role of NCZ to CZ ratio as a parameter providing the optimal clozapine-treatment 


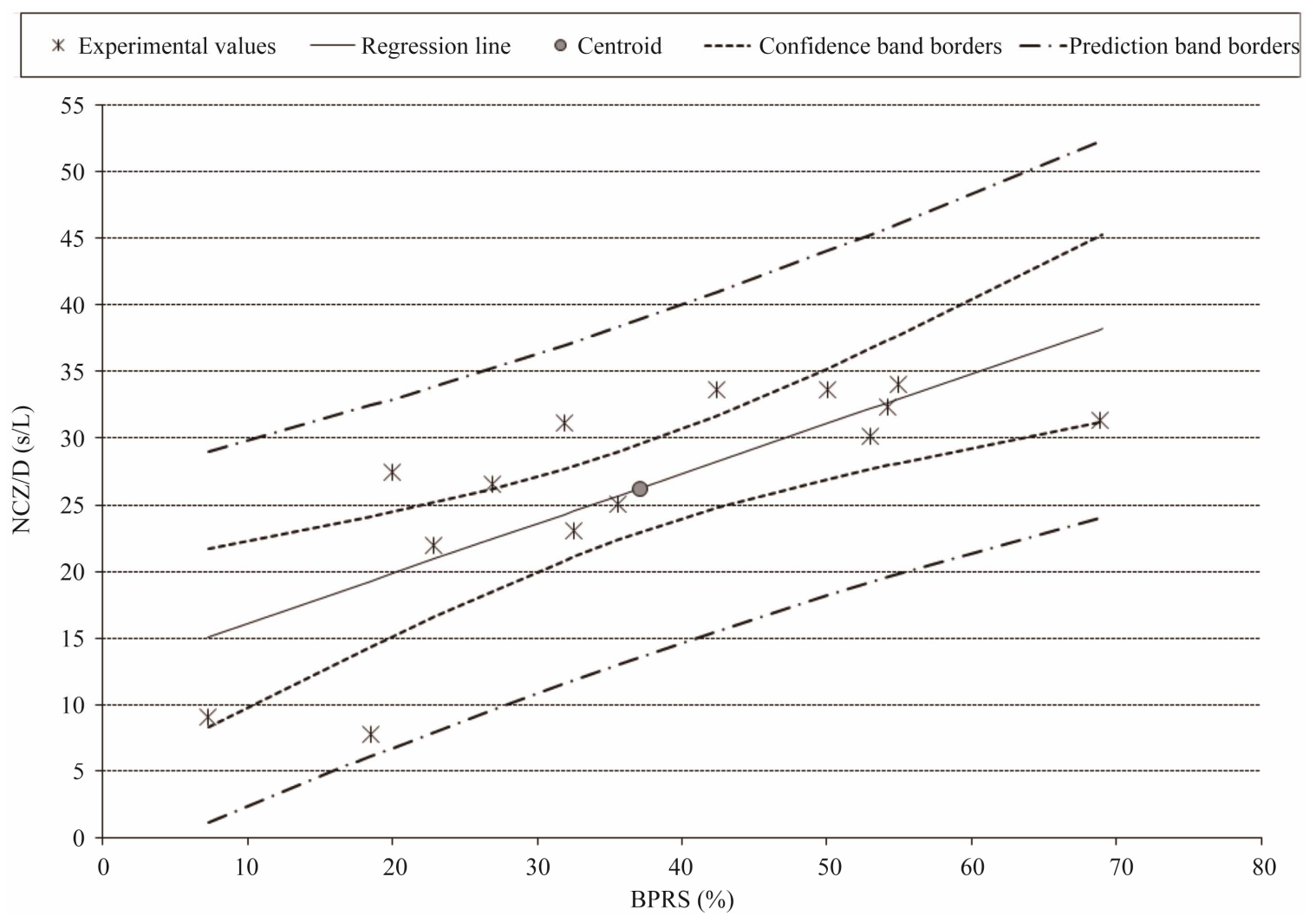

Figure 3. Correlation between plasma norclozapine concentration to clozapine dose ratio (NCZ/D) and clinical response (BPRS). Regression line parameters: $y=12.3+0.38 x$, pReg $<0.0012$, pbo $<0.0056$, pb1 $<0.0012 ; \mathrm{r}^{2}=0.6$ (Determination coefficient); $r=0.77$ (Correlation coefficient). (BPRS) $=[(\mathrm{NCZ} / \mathrm{D})-12.3] / 0.38$ (Prediction equation for the BPRS).

response and those results reported by Palego et al., Jerling et al. and Mauri et al. [24-26] showing that clozapine plasma clearance was not significantly associated with the therapeutic efficiency of the drug.

A significant relationship between the norclozapine plasma concentration to clozapine dose ratio (NCZ/D) and the response to treatment, evaluated by the BPRS levels, was found in this study (Table 4). Our results suggest that the norclozapine plasma concentration to clozapine dose ratio (NCZ/D) could be used as a good indicator for improving the clozapine dose-adaptation strategy.

As a result, this parameter (NCZ/D) appears to be a plasma marker adaptable to a collective scale unlike other parameters (plasma concentration of clozapine, clozapine metabolic ratio) which can only be considered on an individual scale (Table 4).

However, this result, as important as it is, must be taken with caution for at least two reasons. The first relates to the limited number of patients in the sample recruited for this study of the existence of a significant correlation between the NCZ/D relationship and the BPRS score. Validation of such a result would indeed require a greater number of schizophrenics analyzed.
The second reason is about the difficulty of explaining the significance of this relationship from a biological viewpoint. According to its expression, it represents the plasma concentration of norclozapine per unit dose of clozapine administered. But, as a result, it also represents the relationship of the metabolic ratio $(\mathrm{NCZ} / \mathrm{CZ})$ to the plasma clearance of clozapine (D/CZ).

Plasma clearance indirectly brings into play all of the processes involved in the plasma filtration of clozapine. Its catabolism by CYP1A2 assessed by the metabolic ratio constitutes one of these processes. It is conceivable under these conditions that the NCZ/D relationship which also represents the relationship of the metabolic ratio to the clearance, allows the assessment of the relative importance of the filtration processes of clozapine with the exception of the metabolic filtration of CYP1A2. The NCZ/D relationship would be as low for a given metabolic ratio, as the filtration processes involved (with the exception of those relating to CYP1A2) are important.

It seems possible that the negative results observed in our study, particularly the association between the concentration of drug in plasma and its effects on the improvement of the psychopathologic state, may be related 
Table 4. Association between measured parameters and clinical health improvement in 14 schizophrenic patients.

\begin{tabular}{|c|c|c|}
\hline Measured parameters & $\begin{array}{l}\text { Clinical health } \\
\text { improvement }\end{array}$ & Comments/recommendations \\
\hline $\begin{array}{l}\text { Clozapine plasma } \\
\text { concentration }\end{array}$ & BPRS & $\begin{array}{l}\text { There is no correlation between these two parameters. However, plasma concentration of } \\
\text { clozapine appears to be an important parameter, both to individually assess the therapeutic } \\
\text { interval thresholds at the start of treatment and also to ensure medical follow-up relative to } \\
\text { the regularity of taking medications. }\end{array}$ \\
\hline $\begin{array}{l}\text { Clozapine metabolic } \\
\text { ratio }(\mathrm{NCZ} / \mathrm{CZ})\end{array}$ & BPRS & $\begin{array}{l}\text { There is no correlation between the metabolic ratio values and the BPRS scores. It is noted, } \\
\text { however, that patient (a) is the only one whose ratio value plummeted, showing CYP1A2 } \\
\text { enzyme activity of almost zero. It must be noted that not only is the BPRS score of this } \\
\text { patient the lowest ( }(7.3 \%) \text {, but it is also the only patient who developed severe toxicity } \\
\text { expressed by the appearance of agranulocytosis leading to the termination of clozapine } \\
\text { treatment. Overall, if our results show that the metabolic ratio value of clozapine is not a } \\
\text { marker assessing therapeutic efficacy, they nevertheless suggest that a ratio of almost zero } \\
\text { related to very low CYP1A2 activity may constitute a risk factor of developing severe } \\
\text { toxicities during clozapine treatment. }\end{array}$ \\
\hline $\begin{array}{l}\text { Clozapine clearance } \\
\text { (D/CZ) }\end{array}$ & BPRS & $\begin{array}{l}\text { Our results indicate that plasma clozapine clearance cannot constitute a standard marker for } \\
\text { assessment of the therapeutic efficacy of clozapine. }\end{array}$ \\
\hline NCZ/D & BPRS & $\begin{array}{l}\text { There was a significant correlation between clinical health improvement, given by BPRS } \\
\text { scores, and norclozapine plasma concentration to daily clozapine dose ratio (NCZ/D). Such } \\
\text { a result is important insofar as it constitutes an attempt at establishing therapeutic standards } \\
\text { based on clozapine. It is in fact the relationship of the metabolic ratio to the clozapine } \\
\text { clearance. Our results show that the therapeutic efficacy of the drug would be as great as } \\
\text { the value of this parameter is high and no matter which patient is being considered. }\end{array}$ \\
\hline
\end{tabular}

to the small sample size of the study and the heterogeneity of the recruited patients - the majority of our population consists of women. It was reported that sex may have a great influence on health psychotherapeutic improvement [8].

Moreover, it has been proposed that there is a close association between the genetic polymorphism of various genes involved in the metabolism and receptor regulation of anti-psychotic drugs.

So in future investigations, a large and homogeneous sample is needed to characterize in more detail the relationship between clozapine pharmacokinetic parameters and their antipsychotic effects, in order to explain the wide variation in clozapine-treatment responses observed in schizophrenic patients.

\section{ACKNOWLEDGEMENTS}

The authors would like to thank all the individuals who participated in this study.

\section{REFERENCES}

[1] Preskorn, S.H. (2005) Comments on the role of therapeutic drug monitoring for clozapine. Journal of Psychiatric Practice, 11, 340-343. doi:10.1097/00131746-200509000-00006

[2] Kronig, M.H., Munne, R.A., Szymanski, S., Safferman, A.Z., Pollack, S., Cooper, T., Kane, J.M. and Lieberman, J.A. (1995) Plasma clozapine levels and clinical response for treatment-refractory schizophrenic patients. American Journal of Psychiatry, 152, 179-182.

[3] Schulte, P.F.J. (2003) What is an adequate trial with clozapine? Therapeutic drug monitoring and time to response in treatement-refractory schizophrenia. Clinical Pharmacokinetics, 42, 607-618. doi:10.2165/00003088-200342070-00001

[4] Chung, C. and Remington, G. (2005) Predictors and markers of clozapine response. Psychopharmacology, 179, 317335. doi:10.1007/s00213-005-2174-X

[5] Liu, H.C., Chang, W.H., Wei, F.C., Lin, S.K. and Jann M.W. (1996) Monitoring of plasma clozapine levels and its metabolites in refractory schizophrenic patients. Therapeutic Drug Monitoring, 18, 200-207. doi:10.1097/00007691-199604000-00015

[6] Perry, P.J., Miller, D.D., Arndt, S.V. and Cadoret, R.J. (1991) Clozapine and norclozapine plasma concentrations and clinical response of treatment-refractory schizophrenic patients. American Journal of Psychiatry, 148, 231-235.

[7] Chong, S.A., Tan, C.H., Khoo, Y.M., Lee, H.S., Wong, K.E., Ngui, F. and Winslow, M. (1997) Clinical evaluation and plasma clozapine concentrations in Chinese patients with schizophrenia. Therapeutic Drug Monitoring, 19, 219-223. doi:10.1097/00007691-199704000-00018

[8] Yang, F.D., Wang, X.Q., Liu, X.P., Zhao, K.X., Fu, W.H., Hao, X.R., Zhang, X.L., Huang, G.S., Qu, S.C., Bai, J.S., Huang, X.F., Kosten, T.R. and Zhang, X.Y. (2001) Sex difference in QTc prolongation in chronic institutionalized patients with schizophrenia on long-term treatment with typical and atypical antipsychotics. Psychopharmacology, 216, 9-16. doi:10.1007/s00213-011-2188-5

[9] Spina, E., Avenoso, A., Facciolà, G., Scordo, M.G., Ancione, M., Madia, A.G., Ventimiglia, A. and Perucca, E. (2000) Relationship between plasma concentrations of clozapine and norclozapine and therapeutic response in patients with schizophrenia resistant to conventional neuroleptics. Psychopharmacology, 148, 83-89. 


\section{doi:10.1007/s002130050028}

[10] American Psychiatric Association (1994) Diagnostic and statistical manual for mental disorders. 4th Edition, American Psychiatric Press, Washington DC.

[11] Vanelle, J.M. and Amalric, I. (1994) Schizophrénies résistantes. In: EMC Psychiatrie, Elsevier, Paris.

[12] Shen, Y.L., Wu, H.L., Ko, W.K. and Wu, S.M. (2002) Simultaneous determination of clozapine, clozapine $\mathrm{N}$-oxide, $\mathrm{N}$-desmethylclozapine, risperidone, and 9-hydroxyrisperidone in plasma by high performance liquid chromatography with ultraviolet detection. Analytica Chimica Acta, 460, 201-208. doi:10.1016/S0003-2670(02)00239-8

[13] Guitton, C., Abbar, M., Kinowski, J.M., Chabrand, P. and Bressolle, F. (1998) Multiple-dose pharmacokinetics of clozapine in patients with chronic schizophrenia. Journal of Clinical Psychopharmacology, 18, 470-476. doi:10.1097/00004714-199812000-00010

[14] Miller, D.D. (1996) The clinical use of clozapine plasma concentrations in the management of treatment-refractory schizophrenia. Annual Clinical Psychiatry, 8, 99-109. doi:10.3109/10401239609148808

[15] Haring, C., Fleishacker, W.W., Schett, P., Humpel, C., Barnas, C. and Saria, A. (1990) Influence of patient-related variables on clozapine plasma concentrations. American Journal of Psychiatry, 147, 1471-1475.

[16] Edge, S.C., Markovitz, J.S. and DeVane, C.L. (1997) Clozapine drug-drug interactions: A review of the literature. Human Psychopharmacology, 12, 5-20. doi:10.1002/(SICI)1099-1077(199701/02)12:1<5::AID-H UP831>3.0.CO;2-4

[17] Freeman, D.J. and Oyewumi, L.K. (1997) Will routine therapeutic drug monitoring has a place in clozapine therapy? Clinical Pharmacokinetics, 32, 93-100. doi:10.2165/00003088-199732020-00001

[18] Papetti, F., Morel-Pingault, V., Buisse, V., Maziere, L., Banayan, M., Thauby, S., Besnard, T., Darcourt, G. and Pringuey, D. (2007) Clozapine-resistant schizophrenia related to an increased metabolism and benefit of fluvoxamine: Four case reports. Encephale, 33, 811-818. doi:10.1016/j.encep.2007.01.005

[19] Olesen, O.V., Thomsen, K., Jensen, P.N., Rosenberg, R., Wulff, C.H., Rasmussen, N.A., Refshammer, C., Bysted, M., Sørensen, J. and Christensen, J. (1995) Clozapine se- rum levels and side effects during steady state treatment of schizophrenic patients: A cross-sectional study. Psychopharmacology, 117, 371-378. doi:10.1007/BF02246112

[20] Dettling, M., Sachse, C., Brockmöller, J., Schley, J., MüllerOerlinghausen, B., Pickersgill, I., Rolfs, A., Schaub, R.T. and Schmider, J. (2000) Long-term therapeutic drug monitoring of clozapine and metabolites in psychiatric in and out patients. Psychopharmacology, 152, 80-86. doi:10.1007/s002130000503

[21] Llorca, P.M., Lancon, C., Disdier, B., Farisee, J., Sapin, C. and Auquier, P. (2002) Effectiveness of clozapine in neuroleptic-resistant schizophrenia: Clinical response and plasma concentrations. Journal of Psychiatry Neuroscience, 27, 30-37.

[22] Wong, J.O., Leung, S.P., Mak, T., Ng, R.M., Chan, K.T., Cheung, H.H.K., Choi, W.K., Lai, J. and Tsang, A.W.K. (2006) Plasma clozapine levels and clinical response in treatment-refractory Chinese schizophrenic patients. Progress in Neuro-psychopharmacology and Biological Psychiatry, 30, 251-264. doi:10.1016/j.pnpbp.2005.10.008

[23] Doude van Troostwijk, L.J.A.E., Koopmans, R.P., Vermeulen, H.D.B. and Guchelaar, H.J. (2003) CYP1A2 activity is an important determinant of clozapine dosage in schizophrenic patients. European Journal of Pharmacology Science, 20, 451-457. doi:10.1016/j.ejps.2003.09.010

[24] Palego, L., Biondi, L., Giannaccini, G., Sarno, N., Elmi, S., Ciapparelli, A., Cassano, G.B., Lucacchini, A., Martini, C. and Dell’Osso, L. (2002) Clozapine, norclozapine plasma levels, their sum and ratio in 50 psychotic patients: Influence of patient-related variables. Progress in Neuropsychopharmacology and Biological Psychiatry, 26, 473480. doi:10.1016/S0278-5846(01)00291-3

[25] Jerling, M., Merlé, Y., Mentré, F. and Mallet, A. (1997) Population pharmacokinetics of clozapine evaluated with the nonparametric maximum likelihood method. British Journal of Clinical Pharmacology, 44, 447-453. doi:10.1046/j.1365-2125.1997.t01-1-00606.x

[26] Mauri, M.C., Volonteri, L.S., Colasanti, A., Fiorentini, A., De Gaspari, I.F. and Bareggi, S.R. (2007) Clinical pharmacokinetics of atypical antipsychotics: A critical review of the relationship between plasma concentrations and clinical response. Clinical Pharmacokinetics, 46, 359-388. doi:10.2165/00003088-200746050-00001 